\title{
Robust Adaptive Switching Fault-Tolerant Control of a Class of Uncertain Systems against Actuator Faults
}

\author{
Xiao-Zheng Jin \\ The Key Laboratory of Manufacturing Industrial Integrated Automation, Shenyang University, Shenyang Liaoning 110044, China \\ Correspondence should be addressed to Xiao-Zheng Jin; jin445118@163.com
}

Received 18 August 2013; Accepted 16 October 2013

Academic Editor: Bo-Chao Zheng

Copyright ( 2013 Xiao-Zheng Jin. This is an open access article distributed under the Creative Commons Attribution License, which permits unrestricted use, distribution, and reproduction in any medium, provided the original work is properly cited.

\begin{abstract}
This paper deals with the fault-tolerant control (FTC) problem for a class of linear time-invariant systems with time-varying actuator faults and uncertainties. For more general consideration, the faults and uncertainties are supposed to depend on the states of systems and unknown constant bounds. For the sake of eliminating the effects of such state-dependent faults and uncertainties automatically, a switching control strategy which is formulated by a sign function is designed to configure controller based on system's states. And some adjustable control parameters are updated via designing adaptive laws. Based on the information from switching function and the adaptive estimation mechanism, the robust adaptive controllers are constructed to compensate for the effects of faults and uncertainties. Through Lyapunov functions and adaptive schemes, the asymptotic stability of the resulting adaptive FTC uncertain system can be achieved. The effectiveness of the proposed design is illustrated via a rocket fairing structural-acoustic model.
\end{abstract}

\section{Introduction}

In recent years, the safety, reliability, and validity of practical systems have attracted growing attention. However, during the operation of systems, the occurrence of some critical and unpredictable faults of system components, especially in actuators and sensors, is unavoidable and intolerable. As we know, actuators afford the operating function of the whole system. In the event of an actuator fault, the traditional feedback control design may result in unsatisfactory performance of systems or even cause other catastrophic consequence. Thus, fault-tolerant control (FTC) designs are necessary to make the system have capability of tolerating potential actuator faults and to improve the safety and reliability of systems.

In the existing literatures, there are many valuable research results on fault-tolerant control designs in time-delay systems [1, 2], uncertain systems [3-9], nonlinear systems $[10,11]$, network control systems $[12,13]$, and so forth. Among those studies, the FTC design approaches can be generally classified into two types, that is, the passive FTC approach and the active FTC approach. By considering faults as special strong uncertainties without any access of on-line fault information, the passive FTC approach just designs a fixed controller to compensate for the faults and take no additional actions in response to the faults (see, e.g., [14, 15]). Contrarily, active FTC methodologies design adjustable controllers on line and take some response actions to the faults for eliminating the effects of faults. On one hand, the active FTC methods based on fault detection and isolation (FDI) technique can reconfigure or reconstruct controllers by using the real-time fault information provided by the fault diagnosis mechanism [16-18]. But note that some delays of the response action may occur in the procedure of diagnosing the faults, and incorrect decision of FDI mechanism may also happen due to some unexpected factors such as exogenous disturbances and system uncertainties. On the other hand, the active FTC methods based on adaptive technique can regulate controller inputs immediately according to the state changes caused by faults. However, estimating or adjusting parameters by adaptive laws will consume large computation resource, which will make its unavailable in a practical system. Overall, despite the fact that an active FTC approach always causes some drawbacks, it is less conservative and more flexible in dealing with various faults than a passive FTC approach.

Recently, as adaptive technique has capability of quick and automatic response for estimating unknown parameters at each instant, there has been a growing interest in designing active FTC schemes for systems based on adaptive methods. 
The faults of loss actuator effectiveness and parameterizable stuck-actuator faults were studied in [19-24] by using indirect adaptive and direct adaptive methods, respectively. Afterward, the unparameterizable stuck-actuator faults were, respectively, dealt with in $[25,26]$ with indirect adaptive and direct adaptive methods. The problem of bias-/stuck-actuator fault compensation was addressed in $[27,28]$ via an adaptive sliding-mode design with $L_{2}$ gain performance specification. Moreover, for the loss actuator effectiveness faults, the papers $[29,30]$ combined adaptive technique and linear matrix inequality (LMI) technique to provide the optimization of adaptive $H_{\infty}$ performance of an FTC system via dynamic output feedback and state feedback designs, respectively. Using the notion of adaptive $H_{\infty}$ performance in $[29,30]$, the recent papers $[31,32]$ gave less conservative methods with mode-dependent Lyapunov functions against unparameterizable stuck-actuator faults. In terms of the above studies, the adaptive methods can deal with the actuator faults covering loss of effectiveness, outage, and bias-/stuck-actuator faults. Motivated by the effectiveness and real-timely of eliminating a variety of actuator faults, we design a novel active FTC methodology based on adaptive technique to automatically accommodate faults in this paper.

It should be mentioned that the compensation of uncertainties have not been fully considered in the existing robust FTC literature, though some works considered the robust fault-tolerant compensation control with external disturbances in $[25,26]$. Similar to the studies of some related uncertain systems [33], the uncertainties can always be addressed by the LMI technique $[3,4]$ and sidling mode control methods [5] in FTC systems. In [6], an adaptive method was proposed to deal with norm bounded uncertainties and actuator faults. Combining the effects of parametric uncertainties, external disturbances, actuator failures, and control input constraints, the authors of [7] developed fuzzy logic and back-stepping techniques to construct an adaptive controller for achieving high attitude performance of aircraft. For uncertain nonlinear systems, the papers $[8,9]$ utilized Hamilton-Jacobi-inequality-based approach and adaptive diagnostic-based approach to solve the FTC problem, respectively. In those studies, the uncertainties were always considered as norm bounded, sector-bounded, and polytopic-type uncertainties. However, in particular, system's uncertainties may vary along with the changes of system's state, and it will cause huge damage to the systems when the states have big amplitude. Thus, the kind of state-dependent uncertainties should also receive considerable attention.

In this paper, a novel switching adaptive method is proposed to solve the robust fault-tolerant control compensation problem of a class of uncertain linear systems. Similar to the adaptive controllers designed in $[25,26]$, the proposed adaptive approach can also be used for the general actuator fault model, which covers the cases of normal operation, loss of effectiveness, outage, and unparameterizable bias/stuck faults. But different from $[25,26]$, a more general statedependent unparameterizable bias/stuck fault is considered in this paper. Moreover, the state-dependent uncertainties are also addressed in the adaptive controller designs. Here, each control effectiveness and bias/stuck faults are assumed to be unknown, and the rate of dependency of states in uncertainties also needs not to be known. But the maximum degree of dependency states is supposed to be known. Thus, a switching control strategy which is formulated by a sign function is proposed, and, furthermore, some adaptation laws are designed to estimate the unknown controller parameters on line. Based on the switching function and the updated values of these estimations, a class of state feedback controllers is constructed to solve the active FTC problem. Based on the Lyapunov stability theory, the adaptive closed-loop system can be guaranteed to be asymptotically stable in the presence of failures on actuators and uncertainties.

The rest of the paper is organized as follows. The robust fault-tolerant control problem formulation is described in Section 2. In Section 3, the switching adaptive state feedback controllers are developed. Section 4 gives a numerical example of rocket fairing structural-acoustic model and its simulation results. Finally, conclusion is given in Section 5.

\section{Preliminaries and Problem Statement}

In this paper, we consider that a linear time-invariant uncertain continuous-time model captured the following statespace equation:

$$
\dot{x}(t)=\left(A+\Delta_{A}\right) x(t)+\left(B+\Delta_{B}\right) u(t),
$$

where $x(t) \in R^{n}$ is the state, $u(t) \in R^{m}$ is the control input, $A$, $B$ are known real constant matrices with appropriate dimensions, and $\Delta_{A}$ and $\Delta_{B}$ stand for the system uncertainties described by some bounded nonlinear functions.

Similar to [25], we consider the following mathematic model to formulate actuator faults:

$$
u_{i j}^{F}(t)=\rho_{i}^{j}(t) u_{i}(t)+\sigma_{i}^{j} u_{s i}(t), \quad i=1, \ldots, m, j=1, \ldots, L,
$$

where the index $i$ denotes the $i$ th actuator, $j$ stands for the $j$ th faulty mode, $L$ is the total faulty modes, $u_{i j}^{F}(t)$ represents the signal from the $i$ th actuator that has failed in the $j$ th faulty mode, $\rho_{i}^{j}(t)$ is the unknown time-varying actuator efficiency factor, $\underline{\rho}_{i}^{j}$ and $\bar{\rho}_{i}^{j}$ represent the known lower and upper bounds of $\rho_{i}^{j}(t)$, respectively, satisfying $0 \leq \underline{\rho}_{i}^{j} \leq \rho_{i}^{j} \leq$ $\bar{\rho}_{i}^{j} \leq 1$ according to the practical case, and $\sigma_{i}^{j}$ is an unknown constant defined as

$$
\sigma_{i}^{j}= \begin{cases}0, & \rho_{i}^{j}>0, \\ 0 \text { or } 1, & \rho_{i}^{j}=0 .\end{cases}
$$

$u_{s i}(t)$ is the unparametrizable time-varying stuck-actuator fault in the ith actuator satisfying

$$
\left\|u_{s}(t)\right\| \leq \alpha\|x(t)\|+\bar{u}_{s}
$$

where $\alpha \geq 0$ and $\bar{u}_{s}>0$ are unknown constants. Note that the fault model can formulate the faults of actuator outage, loss of effectiveness, and bias and stuck (please see [25] for detail). 
Remark 1. According to the practical case, the occurrence of time-varying unparametrizable faults may be related to the current system states. Thus, we assume that the unparametrizable stuck fault in (4) depends on system's states and a positive constant. Obviously, it is a more general assumption on actuator-stuck faults than the norm-bounded unparametrizable stuck faults considered in [25] or parametrizable stuck faults addressed in [21-24]. Setting $\alpha=0$, the condition reduces to the assumption proposed in [25].

Here, we define the following sets:

$$
\begin{gathered}
\Delta_{\rho^{j}}=\left\{\rho^{j}(t): \rho^{j}(t)=\underset{i=1}{\operatorname{diag}}\left[\rho_{i}^{j}(t)\right], \rho_{i}^{j}(t) \in\left[\underline{\rho}_{i}^{j}, \bar{\rho}_{i}^{j}\right]\right\}, \\
N_{\rho^{j}}=\left\{\rho^{j}(t): \rho^{j}(t)=\operatorname{diag}_{i=1}^{m}\left[\rho_{i}^{j}(t)\right],\right. \\
\left.\rho_{i}^{j}(t)=\underline{\rho}_{i}^{j} \text { or } \rho_{i}^{j}(t)=\bar{\rho}_{i}^{j}\right\},
\end{gathered}
$$

where $i=1,2, \ldots, m, j=1,2, \ldots, L$, and the notation $\operatorname{diag}_{i=1}^{m}\left[\rho_{i}^{j}(t)\right]$ denotes the block-diagonal matrix with $\rho_{i}^{j}(t)$, $i=1,2, \ldots, m$ along the diagonal. And, for the sake of convenience description, for all possible faulty modes $L$, the following uniform actuator fault model is exploited:

$$
u^{F}(t)=\rho(t) u(t)+\sigma u_{s}(t)
$$

where $\rho(t)=\operatorname{diag}\left[\rho_{1}(t), \ldots, \rho_{m}(t)\right] \in\left\{\rho^{1}(t), \ldots, \rho^{L}(t)\right\}$.

Taking actuator faults (6) into consideration, the actual control inputs $u(t)$ generated by actuators are

$$
u^{F}(t)=u(t)+P(t-T)\left[(\rho(t)-I) u(t)+\sigma u_{s}(t)\right],
$$

where $P(t-T)=\operatorname{diag}_{i=1}^{m}\left[p_{i}\left(t-t_{i}\right)\right]$ with $T=\left[t_{1}, t_{2}, \ldots, t_{n}\right]^{T} \in$ $R^{n}$ denotes the time profiles of faults and $t_{i}, i=1,2, \ldots, m$ is the unknown fault-occurrence time representing the time profile of a fault affecting the $i$ th actuator. Then, the faults with time profiles are modeled as follows:

$$
p_{i}\left(t-t_{i}\right)= \begin{cases}0, & \text { if } t<t_{i} \\ 1-e^{-a_{i}\left(t-t_{i}\right)}, & \text { if } t \geq t_{i}\end{cases}
$$

where the scalar $a_{i}>0$ denotes the unknown fault evolution rate. Small values of $a_{i}$ characterize slowly developing faults, also known as incipient faults. For large values of $a_{i}$, the time profile $p_{i}$ approaches a step function that models abrupt faults. It was worth mentioning that the fault time profile described by (8) denotes only the developing speed of a fault, whereas all its other basic features are defined by the vector $(\rho(t)-I) u(t)+\sigma u_{s}(t)$.

Hence, considering actuator faults (7), the dynamics of system (1) can be described by

$$
\begin{aligned}
\dot{x}(t)= & \left(A+\Delta_{A}\right) x(t) \\
& +\left(B+\Delta_{B}\right)(I+P(t-T)(\rho(t)-I)) u(t) \\
& +\left(B+\Delta_{B}\right) P(t-T) \sigma u_{s}(t) .
\end{aligned}
$$

Here, we consider the case of state-feedback fault-tolerant controller design. Thus, we assume that all the states of system are available at every instant and all pairs $\{A, B \rho(t)\}$, are uniformly completely controllable for any actuator failure mode $\rho(t) \in\left\{\rho^{1}(t), \ldots, \rho^{L}(t)\right\}$. Moreover, according to the study of [25], for completely compensating the unparametrizable stuck faults, $\operatorname{rank}[B \rho(t)]=\operatorname{rank}[B]$ should be satisfied for any actuator failure mode $\rho(t) \in\left\{\rho^{1}(t), \ldots, \rho^{L}(t)\right\}$. On the other hand, for an uncertain system (1), the following assumption in FTC design is also assumed to be valid.

Assumption 2. For any vector $x \in R^{n}$, there exists a positive constant $\eta<1$ such that

$$
\left\|x^{T} \Delta_{B}\right\| \leq \eta\left\|x^{T} B \underline{\rho}\right\|,
$$

where $\underline{\rho}=\min \left(\operatorname{diag}_{i=1}^{m}\left[\underline{\rho}_{i}^{j}\right]\right) \in \Delta_{\rho^{j}}, i=1,2, \ldots, m, j=1$, $2, \ldots, \bar{L}$. Moreover, $\Delta_{A}$ is a norm bounded matrix satisfying

$$
\left\|\Delta_{A}\right\| \leq \delta_{A},
$$

where $\delta_{A}$ is an unknown positive constant.

Then, the main objective of this paper is to construct a robust adaptive state feedback controller $u(t)$ such that the closed-loop system (9) can be guaranteed to be asymptotically stable even in the cases of actuator failures and uncertainties.

\section{Switching Robust Adaptive FTC System Design}

For the sake of eliminating the effects of actuator faults and uncertainties completely, a switching adaptive control strategy is proposed in this section. Some adaptive laws are designed to construct the controller with the estimation signals of the unknown actuator failure parameters and upper bound of uncertainties. Then, the asymptotically stable results of the closed-loop FTC system via state feedback are presented in Theorem 3 .

Consider a linear time-invariant uncertain FTC model described by (9) and controller model

$$
u(t)=-\operatorname{sgn}\left(x^{T} B\right)^{T}\left(\widehat{k}_{1}(t)+\widehat{k}_{2}(t)\left\|x^{T} B \underline{\rho}\right\|\right),
$$

where the sign function $\operatorname{sgn}\left(x^{T} B\right)^{T}=\left[\operatorname{sgn}\left(b_{1}\right), \operatorname{sgn}\left(b_{2}\right), \ldots\right.$, $\left.\operatorname{sgn}\left(b_{m}\right)\right]^{T}, b_{i}, i=1,2, \ldots, m$ is the $i$ element of the vector $x^{T} B$ and $\operatorname{sgn}\left(b_{i}\right)$ is defined by

$$
\operatorname{sgn}\left(b_{i}\right)= \begin{cases}-1, & \text { if } b_{i}<0, \\ 1, & \text { if } b_{i}>0, \\ 0, & \text { if } b_{i}=0\end{cases}
$$

$\widehat{k}_{1}(t) \in R$ is updated by the following adaptive law:

$$
\frac{d \widehat{k}_{1}(t)}{d t}=\gamma(1-\eta)\left\|x^{T} B \underline{\rho}\right\|,
$$


where $\gamma$ is any positive constant and $\widehat{k}_{1}\left(t_{0}\right)$ is finite, and, from (14), we can see that $\widehat{k}_{1}(t) \geq 0$ if $\widehat{k}_{1}\left(t_{0}\right) \geq 0 ; \widehat{k}_{2}(t) \in R$ is updated by the following adaptive law:

$$
\frac{d \widehat{k}_{2}(t)}{d t}=\kappa(1-\eta) \lambda_{\min }\|x\|^{2}
$$

where $\kappa$ is any positive constant, $\lambda_{\min }$ is the smallest eigenvalue of $B \rho(B \rho)^{T}$, and $\widehat{k}_{2}\left(t_{0}\right)$ is finite, and, from (15), we can see that $\widehat{k}_{2}(t) \geq 0$ if $\widehat{k}_{2}\left(t_{0}\right) \geq 0$.

Therefore, substituting (12) into (9), the closed-loop FTC system model can be written by

$$
\begin{aligned}
\dot{x}(t)= & \left(A+\Delta_{A}\right) x(t)-B(I+P(t-T)(\rho(t)-I)) \\
& \times \operatorname{sgn}\left(x^{T} B\right)^{T}\left(\widehat{k}_{1}(t)+\widehat{k}_{2}(t)\left\|x^{T} B \underline{\rho}\right\|\right) \\
& -\Delta_{B}(I+P(t-T)(\rho(t)-I)) \\
& \times \operatorname{sgn}\left(x^{T} B\right)^{T}\left(\widehat{k}_{1}(t)+\widehat{k}_{2}(t)\left\|x^{T} B \underline{\rho}\right\|\right) \\
& +\left(B+\Delta_{B}\right) P(t-T) \sigma u_{s}(t) .
\end{aligned}
$$

On the other hand, we denote that

$$
\begin{aligned}
& \widetilde{k}_{1}(t)=\widehat{k}_{1}(t)-k_{1}, \\
& \widetilde{k}_{2}(t)=\widehat{k}_{2}(t)-k_{2} .
\end{aligned}
$$

Since $k_{1}$ and $k_{2}$ are unknown constants, we can write the following error system:

$$
\begin{gathered}
\frac{d \widetilde{k}_{1}(t)}{d t}=\gamma(1-\eta)\left\|x^{T} B \underline{\rho}\right\|, \\
\frac{d \widetilde{k}_{2}(t)}{d t}=\kappa(1-\eta) \lambda_{\min }\|x\|^{2} .
\end{gathered}
$$

In the following, we denote a solution of the closed-loop system and the error system by $\left(x, \widetilde{k}_{1}, \widetilde{k}_{2}\right)(t)$. Then, the following main results can be obtained which shows the globally asymptotic stability of the solutions of the adaptive closedloop system described by (16) and (18).

Theorem 3. Consider the adaptive closed-loop system described by (16) and (18) satisfying Assumption 2. The faulttolerant control system is asymptotically stable for any $\rho(t) \epsilon$ $\Delta_{\rho^{j}}$, if one chooses the controller as (12) and determine $\widehat{k}_{3}(t)$, $\widehat{k}_{4}(t)$ according to the adaptive laws (14) and (15), respectively.

Proof. For the adaptive closed-loop system described by (16), we first define a Lyapunov functional candidate as

$$
V\left(x, \widetilde{k}_{1}, \widetilde{k}_{2}\right)=x^{T} x+\gamma^{-1} \widetilde{k}_{1}^{2}+\kappa^{-1} \widetilde{k}_{2}^{2} .
$$

Then, according to (9), the time derivative of $V$ for $t>0$ associated with a certain failure mode $\rho \in \Delta_{\rho^{j}}$ is

$$
\begin{aligned}
& \frac{d V\left(x, \widetilde{k}_{1}, \widetilde{k}_{2}, t\right)}{d t} \\
& =x^{T}\left[\left(A+\Delta_{A}\right)^{T}+\left(A+\Delta_{A}\right)\right] x \\
& +2 x^{T}\left(B+\Delta_{B}\right)(I+P(t-T)(\rho(t)-I)) u \\
& +2 x^{T}\left(B+\Delta_{B}\right) P(t-T) \sigma u_{s} \\
& +2 \gamma^{-1} \widetilde{k}_{1} \dot{\vec{k}}_{1}+2 \kappa^{-1} \widetilde{k}_{2} \dot{\tilde{k}}_{2} \\
& =2 x^{T}\left(A+\Delta_{A}\right)^{T} x \\
& -2 x^{T} B(I+P(t-T)(\rho(t)-I)) \\
& \times \operatorname{sgn}\left(x^{T} B\right)^{T}\left(\widehat{k}_{1}+\left\|x^{T} B \underline{\rho}\right\| \widehat{k}_{2}\right) \\
& -2 x^{T} \Delta_{B}(I+P(t-T)(\rho(t)-I)) \\
& \times \operatorname{sgn}\left(x^{T} B\right)^{T}\left(\widehat{k}_{1}+\left\|x^{T} B \underline{\rho}\right\| \widehat{k}_{2}\right) \\
& +2 x^{T}\left(B+\Delta_{B}\right) P(t-T) \sigma u_{s} \\
& +2 \gamma^{-1} \widetilde{k}_{1} \dot{\vec{k}}_{1}+2 \kappa^{-1} \widetilde{k}_{2} \dot{\vec{k}}_{2} \text {. }
\end{aligned}
$$

According to the definition of $\rho(t)$ and $P(t-T)$ in $(2)$ and (8), respectively, we know that $(I+P(t-T)(\rho(t)-I))$ is a positive diagonal matrix. Then considering the definition of (13), we have the following fact:

$$
\left\|x^{T} B P_{\rho}\right\| \leq x^{T} B P_{\rho} \operatorname{sgn}\left(x^{T} B\right)^{T},
$$

where $P_{\rho}=I+P(t-T)(\rho(t)-I)$.

Thus, by the light of the inequality of (21) and the condition (4), we can rewrite (20) as

$$
\begin{aligned}
& \frac{d V\left(x, \widetilde{k}_{1}, \widetilde{k}_{2}\right)}{d t} \\
& \leq\|x\|^{2}\left\|A+\Delta_{A}\right\| \\
& -2\left\|x^{T} B P_{\rho}\right\| \widehat{k}_{1}-2\left\|x^{T} B P_{\rho}\right\|\left\|x^{T} B \underline{\rho}\right\| \widehat{k}_{2} \\
& +2\left\|x^{T}\left(B+\Delta_{B}\right)\right\|\|P(t-T) \sigma\|\left\|u_{s}\right\| \\
& +2\left\|x^{T} \Delta_{B} P_{\rho}\right\| \hat{k}_{1}+2\left\|x^{T} \Delta_{B} P_{\rho}\right\|\left\|x^{T} B \underline{\rho}\right\| \hat{k}_{2} \\
& +2 \gamma^{-1} \widetilde{k}_{1} \dot{\tilde{k}}_{1}+2 \kappa^{-1} \widetilde{k}_{2} \dot{\tilde{k}}_{2} \\
& \leq\|x\|^{2}\left\|A+\Delta_{A}\right\| \\
& -2\left\|x^{T} B P_{\rho}\right\| \widehat{k}_{1}-2\left\|x^{T} B P_{\rho}\right\|\left\|x^{T} B \underline{\rho}\right\| \hat{k}_{2} \\
& +2\left\|x^{T}\left(B+\Delta_{B}\right)\right\|\|P(t-T) \sigma\|\left(\alpha\|x(t)\|+\bar{u}_{s}\right)
\end{aligned}
$$




$$
\begin{aligned}
& +2\left\|x^{T} \Delta_{B} P_{\rho}\right\|\left\|\widehat{k}_{1}+2\right\|\left\|x^{T} \Delta_{B} P_{\rho}\right\|\left\|x^{T} B \underline{\rho}\right\| \widehat{k}_{2} \\
& +2 \gamma^{-1} \widetilde{k}_{1} \dot{\tilde{k}}_{1}+2 \kappa^{-1} \widetilde{k}_{2} \dot{\widetilde{k}}_{2} .
\end{aligned}
$$

Due to the fact that $\rho \leq I+P(t-T)(\rho-I) \leq I$, we have $\left\|x^{T} B \rho\right\| \leq\left\|x^{T} \bar{B} P_{\rho}\right\|$ and $\left\|P_{\rho}\right\| \leq 1$. Then, following Assumption 2, we can yield

$$
\begin{aligned}
& \frac{d V\left(x, \widetilde{k}_{1}, \widetilde{k}_{2}\right)}{d t} \\
& \leq\|x\|^{2}\left\|A+\Delta_{A}\right\| \\
& -2(1-\eta)\left\|x^{T} B \underline{\rho}\right\| \widehat{k}_{1}-2(1-\eta)\left\|x^{T} B \underline{\rho}\right\|^{2} \widehat{k}_{2} \\
& +2\left\|x^{T}\left(B+\Delta_{B}\right)\right\|\|P(t-T) \sigma\| \alpha\|x(t)\| \\
& +2\left\|x^{T}\left(B+\Delta_{B}\right)\right\|\|P(t-T) \sigma\| \bar{u}_{s} \\
& +2 \gamma^{-1} \widetilde{k}_{1} \dot{\vec{k}}_{1}+2 \kappa^{-1} \widetilde{k}_{2} \dot{\vec{k}}_{2} \\
& \leq\|x\|^{2}\left\|A+\Delta_{A}\right\| \\
& -2(1-\eta)\left\|x^{T} B \underline{\rho}\right\| \widehat{k}_{1}-2(1-\eta)\left\|x^{T} B \underline{\rho}\right\|^{2} \widehat{k}_{2} \\
& +2\|x\|^{2}\left\|B+\Delta_{B}\right\|\|P(t-T) \sigma\| \alpha \\
& +2\left\|x^{T}\left(B+\Delta_{B}\right)\right\|\|P(t-T) \sigma\| \bar{u}_{s} \\
& +2 \gamma^{-1} \widetilde{k}_{1} \dot{\vec{k}}_{1}+2 \kappa^{-1} \widetilde{k}_{2} \dot{\tilde{k}}_{2} \\
& \leq\|x\|^{2}\left(\left\|A+\Delta_{A}\right\|+2\left\|B+\Delta_{B}\right\|\|P(t-T) \sigma\| \alpha\right) \\
& -2(1-\eta)\left\|x^{T} B \underline{\rho}\right\| \widehat{k}_{1}-2(1-\eta) \lambda_{\text {min }}\|x\|^{2} \widehat{k}_{2} \\
& +2\left\|x^{T}\left(B+\Delta_{B}\right)\right\|\|P(t-T) \sigma\| \bar{u}_{s} \\
& +2 \gamma^{-1} \widetilde{k}_{1} \dot{\widetilde{k}}_{1}+2 \kappa^{-1} \widetilde{k}_{2} \dot{\widetilde{k}}_{2},
\end{aligned}
$$

where $\lambda_{\min }$ is the smallest eigenvalue of $B \rho(B \rho)^{T}$.

On the other hand, according to the definition of (1), (4), and (8), we know that there always exist positive constants $k_{1}$ and $k_{2}$ such that

$$
\begin{aligned}
(1-\eta) & \left\|x^{T} B \underline{\rho}\right\| k_{1} \\
> & \left\|x^{T}\left(B+\Delta_{B}\right)\right\|\|P(t-T) \sigma\| \bar{u}_{s}, \\
(1-\eta) & \lambda_{\min }\|x\|^{2} k_{2} \\
> & \|x\|^{2}\left(\left\|A+\Delta_{A}\right\|+2\left\|B+\Delta_{B}\right\|\|P(t-T) \sigma\| \alpha\right)
\end{aligned}
$$

for any $x \neq 0$.
Then, it follows from (22) that, for any $x \neq 0$,

$$
\begin{aligned}
& \frac{d V\left(x, \widetilde{k}_{1}, \widetilde{k}_{2}\right)}{d t} \\
& <-2(1-\eta)\left\|x^{T} B \underline{\rho}\right\| \widetilde{k}_{1}-2(1-\eta) \lambda_{\min }\|x\|^{2} \widetilde{k}_{2} \\
& \quad+2 \gamma^{-1} \widetilde{k}_{1} \dot{\tilde{k}}_{1}+2 \kappa^{-1} \widetilde{k}_{2} \dot{\tilde{k}}_{2} \\
& =0 .
\end{aligned}
$$

Hence, it is easy to see that $d V\left(x, \widetilde{k}_{1}, \widetilde{k}_{2}\right) / d t<0$ for any $x \neq 0$. Thus, the solutions of closed-loop FTC system are uniformly bounded, and the state $x(t)$ converges asymptotically to zero. This ends the proof.

Theorem 3 indicates that the proposed adaptive switching control scheme can deal with a class of uncertain systems and actuator faults with condition (4) and Assumption 2. Actually, some more relaxant conditions of systems and actuator faults can also be dealt with by the modified adaptive switching control designs. The following theorem is given to illustrate the asymptotic stability results of multidimension state-dependent uncertainties and stuck-actuator faults.

Theorem 4. Consider uncertain system (1) satisfying (10) and

$$
\left\|\Delta_{A}\right\| \leq \delta\|x\|^{p}+\delta_{A},
$$

where $\delta, \delta_{A}>0$, and actuator faults formulated in (6) with

$$
\left\|u_{s}(t)\right\| \leq \alpha\|x(t)\|^{q}+\bar{u}_{s},
$$

where $p$ and $q$ are known maximum degree of $\|x(t)\|$ denoting the rate of dependency of states using the following controller:

$$
\begin{aligned}
u_{i}(t)= & -\operatorname{sgn}\left(x^{T} B\right)^{T} \\
& \times\left(\widehat{k}_{1}(t)+\widehat{k}_{2}(t)\left\|x^{T} B \underline{\rho}\right\|\right. \\
& \left.\quad+\widehat{k}_{3}(t)\left\|x^{T} B \underline{\rho}\right\|^{p+1}+\widehat{k}_{4}(t)\left\|x^{T} B \underline{\rho}\right\|^{q}\right),
\end{aligned}
$$

where $\widehat{k}_{1}(t)$ and $\widehat{k}_{2}(t)$ are updated by adaptive laws (14) and (15) and $\widehat{k}_{3}(t)$ and $\widehat{k}_{4}(t)$ are adjusted by the following adaptive laws:

$$
\begin{gathered}
\frac{d \widehat{k}_{3}(t)}{d t}=\zeta(1-\eta)\|x\|^{p+2}, \\
\frac{d \widehat{k}_{4}(t)}{d t}=\xi(1-\eta) \lambda_{\min }\|x\|^{q+1},
\end{gathered}
$$

where $\zeta$ and $\xi$ are any positive constants. Then, the system states are asymptotically convergence to zero within finite time.

Proof. Similar to the proof of Theorem 3, we first define a Lyapunov functional candidate as:

$$
V\left(x, \widetilde{k}_{1}, \widetilde{k}_{2}, \widetilde{k}_{3}, \widetilde{k}_{4}\right)=x^{T} x+\gamma^{-1} \widetilde{k}_{1}^{2}+\kappa^{-1} \widetilde{k}_{2}^{2}+\zeta^{-1} \widetilde{k}_{3}^{2}+\xi^{-1} \widetilde{k}_{4}^{2} .
$$


Then, in terms of (21), the time derivative of $V$ for $t>0$ associated with a certain failure mode $\rho \in \Delta_{\rho^{j}}$ is

$$
\begin{aligned}
& \frac{d V\left(x, \tilde{k}_{1}, \tilde{k}_{2}, \tilde{k}_{3}, \tilde{k}_{4}, t\right)}{d t} \\
& =x^{T}\left[\left(A+\Delta_{A}\right)^{T}+\left(A+\Delta_{A}\right)\right] x \\
& +2 x^{T}\left(B+\Delta_{B}\right)(I+P(t-T)(\rho(t)-I)) u \\
& +2 x^{T}\left(B+\Delta_{B}\right) P(t-T) \sigma u_{s} \\
& +2 \gamma^{-1} \widetilde{k}_{1} \dot{\widetilde{k}}_{1}+2 \kappa^{-1} \widetilde{k}_{2} \dot{\widetilde{k}}_{2}+2 \zeta^{-1} \widetilde{k}_{3} \dot{\widetilde{k}}_{3}+2 \xi^{-1} \widetilde{k}_{4} \dot{\widetilde{k}}_{4} \\
& \leq 2\|x\|^{2}\left(\|A\|+\left\|\Delta_{A}\right\|\right) \\
& -2\left\|x^{T} B P_{\rho}\right\| \hat{k}_{1}-2\left\|x^{T} B P_{\rho}\right\|\left\|x^{T} B \underline{\rho}\right\| \hat{k}_{2} \\
& -2\left\|x^{T} B P_{\rho}\right\|\left\|x^{T} B \underline{\rho}\right\|^{p+1} \widehat{k}_{3}-2\left\|x^{T} B P_{\rho}\right\|\left\|x^{T} B \underline{\rho}\right\|^{q} \widehat{k}_{4} \\
& +2\left\|x^{T}\left(B+\Delta_{B}\right)\right\|\|P(t-T) \sigma\|\left\|u_{s}\right\| \\
& +2\left\|x^{T} \Delta_{B} P_{\rho}\right\| \hat{k}_{1}+2\left\|x^{T} \Delta_{B} P_{\rho}\right\|\left\|x^{T} B \underline{\rho}\right\| \widehat{k}_{2} \\
& +2\left\|x^{T} \Delta_{B} P_{\rho}\right\|\left\|x^{T} B \underline{\rho}\right\|^{p+1} \widehat{k}_{3}+2\left\|x^{T} \Delta_{B} P_{\rho}\right\|\left\|x^{T} B \underline{\rho}\right\|^{q} \widehat{k}_{4} \\
& +2 \gamma^{-1} \widetilde{k}_{1} \dot{\widetilde{k}}_{1}+2 \kappa^{-1} \widetilde{k}_{2} \dot{\widetilde{k}}_{2}+2 \zeta^{-1} \widetilde{k}_{3} \dot{\widetilde{k}}_{3}+2 \xi^{-1} \widetilde{k}_{4} \dot{\widetilde{k}}_{4} .
\end{aligned}
$$

From the fact of $\left\|x^{T} B \rho\right\| \leq\left\|x^{T} B P_{\rho}\right\|,\left\|P_{\rho}\right\| \leq 1$, and inequalities (10), (26), and ( $\overline{2} 7)$, we can rewrite (31) as

$$
\begin{aligned}
& \frac{d V\left(x, \tilde{k}_{1}, \tilde{k}_{2}, \tilde{k}_{3}, \tilde{k}_{4}, t\right)}{d t} \\
& \leq 2\|x\|^{2}\left(\|A\|+\delta\|x\|^{p}+\delta_{A}\right) \\
& -2(1-\eta)\left\|x^{T} B \underline{\rho}\right\| \widehat{k}_{1}-2(1-\eta)\left\|x^{T} B \underline{\rho}\right\|^{2} \widehat{k}_{2} \\
& -2(1-\eta)\left\|x^{T} B \underline{\rho}\right\|^{p+2} \widehat{k}_{3}-2(1-\eta)\left\|x^{T} B \underline{\rho}\right\|^{q+1} \widehat{k}_{4} \\
& +2\left\|x^{T}\left(B+\Delta_{B}\right)\right\|\|P(t-T) \sigma\|\left(\alpha\|x(t)\|^{q}+\bar{u}_{s}\right) \\
& +2 \gamma^{-1} \widetilde{k}_{1} \dot{\widetilde{k}}_{1}+2 \kappa^{-1} \widetilde{k}_{2} \dot{\vec{k}}_{2}+2 \zeta^{-1} \widetilde{k}_{3} \dot{\vec{k}}_{3}+2 \xi^{-1} \widetilde{k}_{4} \dot{\widetilde{k}}_{4} \\
& \leq 2\|x\|^{2}\left(\|A\|+\delta_{A}\right)+2 \delta\|x\|^{p+2} \\
& -2(1-\eta)\left\|x^{T} B \rho\right\| \widehat{k}_{1}-2(1-\eta)\left\|x^{T} B \rho\right\|^{2} \widehat{k}_{2} \\
& -2(1-\eta)\left\|x^{T} B \underline{\rho}\right\|^{p+2} \widehat{k}_{3}-2(1-\eta)\left\|x^{T} B \underline{\rho}\right\|^{q+1} \widehat{k}_{4} \\
& +2 \alpha\|x\|^{q+1}\left\|\left(B+\Delta_{B}\right)\right\|\|P(t-T) \sigma\| \\
& +2\left\|x^{T}\left(B+\Delta_{B}\right)\right\|\|P(t-T) \sigma\| \bar{u}_{s} \\
& +2 \gamma^{-1} \widetilde{k}_{1} \dot{\widetilde{k}}_{1}+2 \kappa^{-1} \widetilde{k}_{2} \dot{\widetilde{k}}_{2}+2 \zeta^{-1} \widetilde{k}_{3} \dot{\widetilde{k}}_{3}+2 \xi^{-1} \widetilde{k}_{4} \dot{\widetilde{k}}_{4}
\end{aligned}
$$

$$
\begin{aligned}
\leq & 2\|x\|^{2}\left(\|A\|+\delta_{A}\right)+2 \delta\|x\|^{p+2} \\
& -2(1-\eta)\left\|x^{T} B \underline{\rho}\right\| \widehat{k}_{1}-2(1-\eta) \lambda_{\min }\|x\|^{2} \widehat{k}_{2} \\
& -2(1-\eta) \lambda_{\min }\|x\|^{p+2} \widehat{k}_{3}-2(1-\eta) \lambda_{\min }\|x\|^{q+1} \widehat{k}_{4} \\
& +2 \alpha\|x\|^{q+1}\left\|\left(B+\Delta_{B}\right)\right\|\|P(t-T) \sigma\| \\
& +2\left\|x^{T}\left(B+\Delta_{B}\right)\right\|\|P(t-T) \sigma\| \bar{u}_{s} \\
& +2 \gamma^{-1} \widetilde{k}_{1} \dot{\widetilde{k}}_{1}+2 \kappa^{-1} \widetilde{k}_{2} \dot{\widetilde{k}}_{2}+2 \zeta^{-1} \widetilde{k}_{3} \dot{\widetilde{k}}_{3}+2 \xi^{-1} \widetilde{k}_{4} \dot{\widetilde{k}}_{4},
\end{aligned}
$$

where $\lambda_{\text {min }}$ is the smallest eigenvalue of $B \underline{\rho}(B \underline{\rho})^{T}$.

Since $\delta, \alpha, \delta_{A}$, and $\bar{u}_{s}$ are constants, the $\bar{n}$ there always exist positive constants $k_{1}, k_{2}, k_{3}$, and $k_{4}$ such that

$$
\begin{gathered}
(1-\eta)\left\|x^{T} B \underline{\rho}\right\| k_{1}>\left\|x^{T}\left(B+\Delta_{B}\right)\right\|\|P(t-T) \sigma\| \bar{u}_{s}, \\
(1-\eta) \lambda_{\min }\|x\|^{2} k_{2}>\|x\|^{2}\left(\|A\|+\delta_{A}\right), \\
(1-\eta) \lambda_{\min }\|x\|^{p+2} k_{3}>\delta\|x\|^{p+2}, \\
(1-\eta) \lambda_{\min }\|x\|^{q+1} k_{4}>\alpha\|x\|^{q+1}\left\|\left(B+\Delta_{B}\right)\right\|\|P(t-T) \sigma\|
\end{gathered}
$$

for any $x \neq 0$.

Then, it follows from (32) that, for any $x \neq 0$,

$$
\begin{aligned}
& \frac{d V\left(x, \tilde{k}_{1}, \widetilde{k}_{2}, \widetilde{k}_{3}, \widetilde{k}_{4}, t\right)}{d t} \\
& <-2(1-\eta)\left\|x^{T} B \underline{\rho}\right\| \widetilde{k}_{1}-2(1-\eta) \lambda_{\min }\|x\|^{2} \widetilde{k}_{2} \\
& \quad-2(1-\eta) \lambda_{\min }\|x\|^{p+2} \widetilde{k}_{3}-2(1-\eta) \lambda_{\min }\|x\|^{q+1} \widetilde{k}_{4} \\
& \quad+2 \gamma^{-1} \widetilde{k}_{1} \dot{\widetilde{k}}_{1}+2 \kappa^{-1} \widetilde{k}_{2} \dot{\widetilde{k}}_{2}+2 \zeta^{-1} \widetilde{k}_{3} \dot{\widetilde{k}}_{3}+2 \xi^{-1} \widetilde{k}_{4} \dot{\widetilde{k}}_{4} \\
& =0 .
\end{aligned}
$$

Hence, it indicates that, for any $x \neq 0, d V\left(x, \tilde{k}_{1}, \tilde{k}_{2}, \tilde{k}_{3}, \tilde{k}_{4}\right.$, $t) / d t<0$. Thus, the global adaptive fault-tolerant compensation problem of uncertain system is solvable. The solutions of closed-loop FTC system are uniformly bounded, and the state $x(t)$ converges asymptotically to zero.

Remark 5. Theorem 4 has shown that the proposed method can compensate for multidimension state-dependent uncertainties and stuck-actuator faults. However, the dependency rate of states should be known to construct the compensation controllers, which will bring some limitation of this method. On the other hand, inequality (10) is also a conservative condition for uncertainties. Some more effective methods should be investigated to reduce the limitation in future studies.

From Theorems 3 and 4, we know that the multidimension state-dependent uncertainties and unparametrizable stuck-actuator faults can be completely compensated by 
the proposed adaptive switching control method. Actually, the proposed adaptive switching compensation control designs are not only suitable for the system's uncertainties, but also suitable for the controller gain uncertainties. The following corollary is given to illustrate the asymptotic stability of such nonfragile systems in the case of additive controller coefficient variations.

Corollary 6. Consider uncertain system (1) satisfying (10), (26), and (27) using the following controller:

$$
\begin{aligned}
u_{i}(t)=-\operatorname{sgn}\left(x^{T} B\right)^{T} & \left(\widehat{k}_{1}(t)+\Delta_{k 1}+\left(\widehat{k}_{2}(t)+\Delta_{k 2}\right)\left\|x^{T} B \underline{\rho}\right\|\right. \\
& +\left(\widehat{k}_{3}(t)+\Delta_{k 3}\right)\left\|x^{T} B \underline{\rho}\right\|^{p+1} \\
& \left.+\left(\widehat{k}_{4}(t)+\Delta_{k 4}\right)\left\|x^{T} B \underline{\rho}\right\|^{q}\right),
\end{aligned}
$$

where $\Delta_{k 1}, \Delta_{k 2}, \Delta_{k 3}$, and $\Delta_{k 4}$ are bounded additive controller coefficient variations satisfying $\left|\Delta_{k l}\right| \leq \delta_{k l}, \delta_{k l}$ is unknown positive constants, $l=1,2,3,4$, and $\widehat{k}_{1}(t), \widehat{k}_{2}(t), \widehat{k}_{3}(t)$, and $\widehat{k}_{4}(t)$ are updated by adaptive laws (14), (15), and (29). Then, the system states are asymptotically convergence to zero within finite time.

Proof. The proof of this corollary is omitted. It is quite similar to the one of Theorem 4 .

Remark 7. From Theorems 3 and 4, we can see that the effects of system matrix $A$ have also been eliminated in the designs. Different from other designs to construct a Lyapunov matrix $P$ from system matrices $A$ and $B$ in [25], the method needs not to solve the Lyapunov matrix. Thus, the estimation of $K_{1}$ in [25] has also been omitted. And it can reduce the computation burden in the designs. It should also be mentioned that the same design can also be realized by modifying $K_{2}$ such as

$$
K_{2}(t)=\frac{-\left(x^{T} P B\right)^{T} \beta\left\|x^{T} P B\right\|\left(\widehat{k}_{1}(t)+\widehat{k}_{2}(t)\left\|x^{T} P B\right\|\right)}{\left\|x^{T} P B\right\|^{2} \alpha}
$$

in [25]. In other word, the function of (12) and (36) is the same.

Remark 8. Actually, several methods have been proposed to completely compensate for the effects of time-varying matched perturbations and redundant actuator faults (e.g., $[25,26,31])$. All of the methods have a limitation that chattering phenomenon will occur when the states or tracking errors converge to zero. Note that the limitation still exists in the method proposed in this paper, because of the usage of sign function $\operatorname{sgn}(\cdot)$ in (13). The limitation can be reduced by some improved control strategies such as filtering theory, boundary layer around the switching surface, and switching gain which is proposed in sliding mode control technique $[34,35]$. However, a bounded stability result can be achieved rather than asymptotic stability.

\section{Numerical Example}

Similar to $[22,23]$, the rocket fairing structural-acoustic model which is also used in [25] is adopted with the following system matrices:

$$
\begin{gathered}
A=\left[\begin{array}{cccc}
0 & 1 & 0.0802 & 1.0415 \\
-0.1980 & -0.115 & -0.0318 & 0.3 \\
-3.0500 & 1.1880 & -0.4650 & 0.9 \\
0 & 0.0805 & 1 & 0
\end{array}\right], \\
B=\left[\begin{array}{ccc}
1 & 1.55 & 0.75 \\
0.975 & 0.8 & 0.85 \\
0 & 0 & 0 \\
0 & 0 & 0
\end{array}\right] .
\end{gathered}
$$

With the similar simulation of [25], we consider four possible faulty modes as follows.

Normal Mode 1. All the third actuators are normal; that is, $\rho_{i}^{1}=1, \sigma_{i}^{1}=0, a_{i}=0$, and $i=1,2,3$.

Fault Mode 2. The first actuator is outage or stuck, the second and the third actuators may be normal or loss of effectiveness; described by $\rho_{1}^{2}=0, b_{2} \leq \rho_{2}^{2} \leq 1, b_{3} \leq \rho_{3}^{2} \leq 1$, and $b_{2}=0.3$, and $b_{3}=0.5$, which denotes the maximum loss of effectiveness for the second and the third actuators, and $a_{2}=2, a_{3}=3$.

Fault Mode 3. The second actuator is outage or stuck, the first and third actuators may be normal or loss of effectiveness, that is, $\rho_{2}^{3}=0, b_{1} \leq \rho_{1}^{3} \leq 1, b_{3} \leq \rho_{3}^{3} \leq 1, b_{1}=0.5, b_{3}=0.3$, which denotes the maximum loss of effectiveness for the first and the third actuators, and $a_{1}=3, a_{3}=2$.

Fault Mode 4. The third actuator is outage or stuck, the first and second actuators may be normal or loss of effectiveness; that is, $\rho_{3}^{4}=0, c_{1} \leq \rho_{1}^{4} \leq 1, c_{2} \leq \rho_{2}^{4} \leq 1, b_{1}=0.5$, and $b_{2}=0.2$, which denotes the maximum loss of effectiveness for the first and the second actuators, and $a_{1}=1, a_{2}=0.5$.

For the sake of verifying the effectiveness of the proposed adaptive method, the simulations are given with the following parameters and initial conditions:

$$
\begin{aligned}
\gamma=50, \quad \kappa=1, \quad \zeta=10, & \varepsilon=10, \\
x(0)=[0,1,0.5,-1]^{T}, & \widehat{k}_{i}(0)=0, \quad i=1,2,3,4 .
\end{aligned}
$$

The following faulty case is considered in the simulations; that is, before 8 second, the systems operate in normal case and the uncertainties $\Delta_{A}=(-0.3 \sin (0.1 t)\|x\|+0.5) \times A$, $\Delta_{B}=0.2 \times B$ enter into the system at the beginning $(t \geq$ $0)$. At 8 second, the fault mode 2 has occurred; that is, the first actuator has stuck at $u_{s 1}(t)=2+0.5 \sin (0.1 t)-$ $0.5 \cos (0.5 t)\|x\|^{2}$ and the third actuator loss of effectiveness described by $\rho_{3}=1-0.03 t$ until loss effectiveness of $50 \%$.

Figure 1 is the response curves of the system's states with robust adaptive state feedback controller in the abovementioned faulty case. Figure 2 illustrates the estimated curves of controller parameters $\widehat{k}_{1}(t), \widehat{k}_{2}(t), \widehat{k}_{3}(t)$, and $\widehat{k}_{4}(t)$, respectively. It is easy to see that the estimates can converge 




FIgURE 1: Response curves of the system's states $x_{i}(t), i=1,2,3,4$ under the proposed adaptive switching controller (28).



(a)

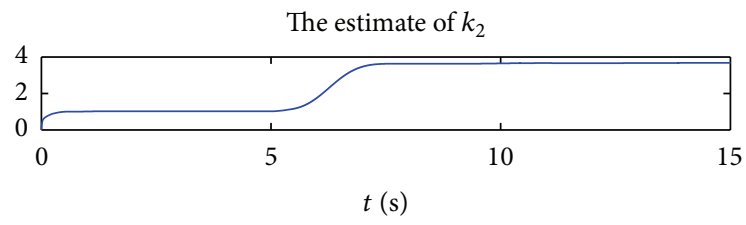

(b)

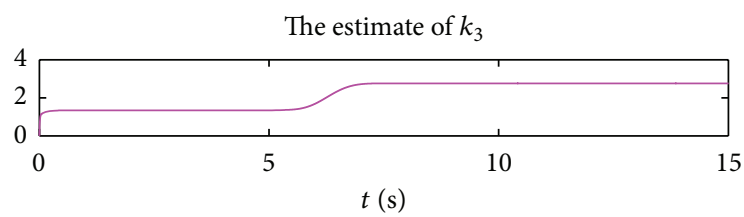

(c)

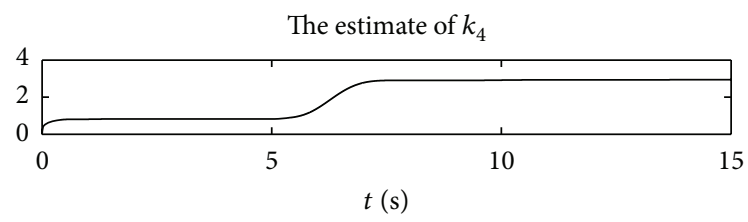

(d)

FIgURE 2: Response curves of the adaptive adjustable control parameters $\widehat{k}_{1}(t), \widehat{k}_{2}(t), \widehat{k}_{3}(t)$, and $\widehat{k}_{4}(t)$, respectively. and the closed-loop FTC system asymptotically stable in the presence of faults on actuators and uncertainties.

\section{Conclusion}

This paper presents a switching adaptive method for robust fault-tolerant control problem of actuator faults and compensation in continuous-time linear systems. The fault model of normal operation, loss of effectiveness, outage, and stuck are considered, and the bias-/stuck-actuator faults and system uncertainties are supposed to be satisfying a state-dependent condition. The switching robust adaptive control schemes are constructed based on a sign function and some updating adaptation laws which are used to estimate the controller parameters on line. The proposed state feedback controllers can automatically compensate the fault and uncertainty effects and guarantee the asymptotically stable of the system. A numerical example has shown the effectiveness of the proposed method.

One of the further research topics is to eliminate the assumption of knowing the dependency rate of states $p$ and $q$ and to reduce the condition of (10). Additionally, the chattering problem should be further considered for the sake of application of the proposed method.

\section{Acknowledgments}

This work is supported by the National Natural Science Foundation (Grant nos. 61104029, 61273155, and 61203087), Program for Liaoning Excellent Talents in University (LNET) (Grant no. LJQ2013122), the science and technology plan of Liaoning Province (2011219011), the Natural Science Foundation of Liaoning Province (Grant no. 201202156), and the Scientific Research Foundation for Doctor of Liaoning Province (Grant no. 20121040).

\section{References}

[1] Z. Mao, B. Jiang, and P. Shi, "Observer-based fault-tolerant control for a class of networked control systems with transfer delays," Journal of the Franklin Institute, vol. 348, no. 4, pp. 763776, 2011.

[2] E. Tian, D. Yue, and C. Peng, "Reliable control for networked control systems with probabilistic actuator fault and random delays," Journal of the Franklin Institute, vol. 347, no. 10, pp. 19071926, 2010.

[3] C. Cheng and Q. Zhao, "Reliable control of uncertain delayed systems with integral quadratic constraints," IEE Proceedings of Control Theory and Applications, vol. 151, no. 6, pp. 790-796, 2004.

[4] S. Ye, Y. Zhang, X. Wang, and B. Jiang, "Fault-tolerant control for a class of uncertain systems with actuator faults," Tsinghua Science and Technology, vol. 15, no. 2, pp. 174-183, 2010.

[5] T. Wang, W. Xie, and Y. Zhang, "Sliding mode fault tolerant control dealing with modeling uncertainties and actuator faults," ISA Transactions, vol. 51, no. 3, pp. 386-392, 2012.

[6] X. J. Li and G. H. Yang, "Robust adaptive fault-tolerant control for uncertain linear systems with actuator failures," IET Control Theory \& Applications, vol. 6, no. 10, pp. 1544-1551, 2012. 
[7] A.-M. Zou and K. D. Kumar, "Adaptive fuzzy fault-tolerant attitude control of spacecraft," Control Engineering Practice, vol. 19, no. 1, pp. 10-21, 2011.

[8] G.-H. Yang, J. L. Wang, and Y. C. Soh, "Reliable guaranteed cost control for uncertain nonlinear systems," IEEE Transactions on Automatic Control, vol. 45, no. 11, pp. 2188-2192, 2000.

[9] X. Zhang, T. Parisini, and M. M. Polycarpou, "Adaptive fault-tolerant control of nonlinear uncertain systems: an information-based diagnostic approach," IEEE Transactions on Automatic Control, vol. 49, no. 8, pp. 1259-1274, 2004.

[10] S. Varma and K. D. Kumar, "Fault tolerant satellite attitude control using solar radiation pressure based on nonlinear adaptive sliding mode," Acta Astronautica, vol. 66, no. 3-4, pp. 486-500, 2010.

[11] Z. Zhang and W. Chen, "Adaptive output feedback control of nonlinear systems with actuator failures," Information Sciences, vol. 179, no. 24, pp. 4249-4260, 2009.

[12] C.-X. Yang, Z.-H. Guan, and J. Huang, "Stochastic fault tolerant control of networked control systems," Journal of the Franklin Institute, vol. 346, no. 10, pp. 1006-1020, 2009.

[13] H. Zhihong, Z. Yuan, and X. Chang, "A robust fault-tolerant control strategy for networked control systems," Journal of Network and Computer Applications, vol. 34, no. 2, pp. 708-714, 2011.

[14] R. J. Veillette, J. B. Medanic, and W. R. Perkins, "Design of reliable control systems," IEEE Transactions on Automatic Control, vol. 37, no. 3, pp. 290-304, 1992.

[15] G.-H. Yang, J. L. Wang, and Y. C. Soh, "Reliable $H_{\infty}$ controller design for linear systems," Automatica, vol. 37, no. 5, pp. 717-725, 2001.

[16] K. Zhang, B. Jiang, and P. Shi, "Fast fault estimation and accommodation for dynamical systems," IET Control Theory and Applications, vol. 3, no. 2, pp. 189-199, 2009.

[17] H. Alwi and C. Edwards, "Fault detection and fault-tolerant control of a civil aircraft using a sliding-mode-based scheme," IEEE Transactions on Control Systems Technology, vol. 16, no. 3, pp. 499-510, 2008.

[18] W. Chen and F. N. Chowdhury, "Analysis and detection of incipient faults in post-fault systems subject to adaptive faulttolerant control," International Journal of Adaptive Control and Signal Processing, vol. 22, no. 9, pp. 815-832, 2008.

[19] J. D. Boskovic and R. K. Mehra, "A robust adaptive reconfigurable flight con-trol scheme for accommodation of control effector failures," in Proceeding of the American Control Conference, vol. 2, pp. 1127-1132, Arlington, Va, USA, 2001.

[20] D. Ye and G.-H. Yang, "Adaptive fault-tolerant tracking control against actuator faults with application to flight control," IEEE Transactions on Control Systems Technology, vol. 14, no. 6, pp. 1088-1096, 2006.

[21] G. Tao, S. M. Joshi, and X. Ma, "Adaptive state feedback and tracking control of systems with actuator failures," IEEE Transactions on Automatic Control, vol. 46, no. 1, pp. 78-95, 2001.

[22] L. F. Wang, B. Huang, and K. C. Tan, "Fault-tolerant vibration control in a networked and embedded rocket fairing system," IEEE Transactions on Industrial Electronics, vol. 51, no. 6, pp. 1127-1141, 2004.

[23] X. Tang, G. Tao, L. Wang, and J. A. Stankovic, "Robust and adaptive actuator failure compensation designs for a rocket fairing structural-acoustic model," IEEE Transactions on Aerospace and Electronic Systems, vol. 40, no. 4, pp. 1359-1366, 2004.
[24] X. D. Tang, G. Tao, and S. M. Joshi, "Adaptive actuator failure compensation for nonlinear MIMO systems with an aircraft control application," Automatica, vol. 43, no. 11, pp. 1869-1883, 2007.

[25] X.-Z. Jin and G.-H. Yang, "Robust adaptive fault-tolerant compensation control with actuator failures and bounded disturbances," Acta Automatica Sinica, vol. 35, no. 3, pp. 305309, 2009.

[26] X. Jin, G. Yang, and Y. Li, "Robust fault-tolerant controller design for linear time-invariant systems with actuator failures: an indirect adaptive method," Journal of Control Theory and Applications, vol. 8, no. 4, pp. 471-478, 2010.

[27] Q. Hu, "Robust adaptive sliding-mode fault-tolerant control with $\mathrm{L}_{2}$-gain performance for flexible spacecraft using redundant reaction wheels," IET Control Theory and Applications, vol. 4, no. 6, pp. 1055-1070, 2010.

[28] Q. Hu and B. Xiao, "Adaptive fault tolerant control using integral sliding mode strategy with application to flexible spacecraft," International Journal of Systems Science, vol. 44, no. 12, pp. 22732286, 2013.

[29] G.-H. Yang and D. Ye, "Adaptive fault-tolerant $H_{\infty}$ control via dynamic output feedback for linear systems against actuator faults," in Proceeding of the 45th IEEE Conference on Decision and Control 2006, pp. 3524-3529, San Diego, Calif, USA, December 2006.

[30] G.-H. Yang and D. Ye, "Reliable $H_{\infty}$ control of linear systems with adaptive mechanism," IEEE Transactions on Automatic Control, vol. 55, no. 1, pp. 242-247, 2010.

[31] X. Z. Jin, G. H. Yang, X. H. Chang, and W. W. Che, "Robust fault-tolerant $H_{\infty}$ control with adaptive compensation," Acta Automatica Sinica, vol. 39, no. 1, pp. 31-42, 2013.

[32] X. Z. Jin, G. H. Yang, and X. H. Chang, "Robust $H_{\infty}$ and adaptive tracking control against actuator faults with a linearised aircraft application," International Journal of Systems Sciences, vol. 44, no. 1, pp. 151-165, 2013.

[33] X. Xie, X. Zhu, and Y. Wang, "Relaxed global asymptotic stability of 2-D state-space digital filters described by Roesser model with polytopic-type uncertainty," Signal Processing, vol. 94, no. 1, pp. 102-107, 2014.

[34] W.-J. Cao and J.-X. Xu, "Nonlinear integral-type sliding surface for both matched and unmatched uncertain systems," IEEE Transactions on Automatic Control, vol. 49, no. 8, pp. 1355-1360, 2004.

[35] P. Kachroo and M. Tomizuka, "Chattering reduction and error convergence in the sliding-mode control of a class of nonlinear systems," IEEE Transactions on Automatic Control, vol. 41, no. 7, pp. 1063-1068, 1996. 


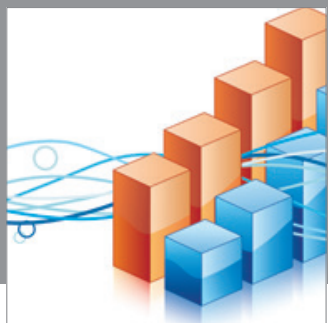

Advances in

Operations Research

mansans



The Scientific World Journal
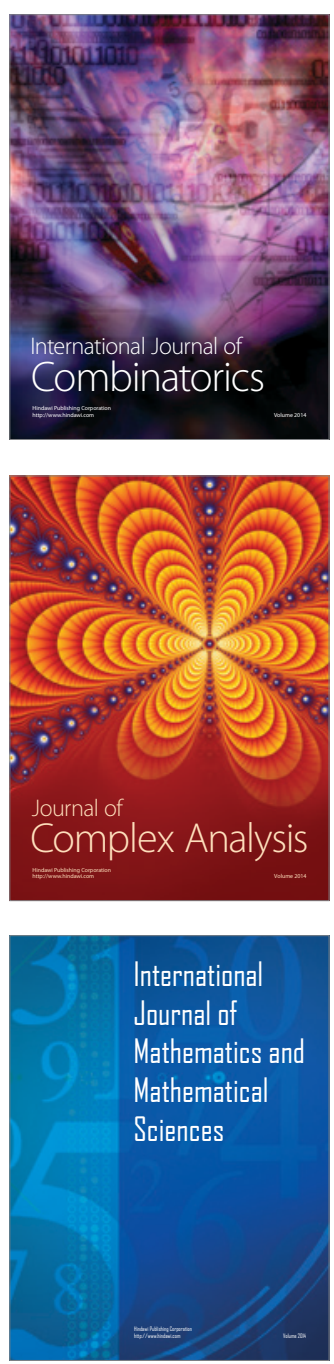
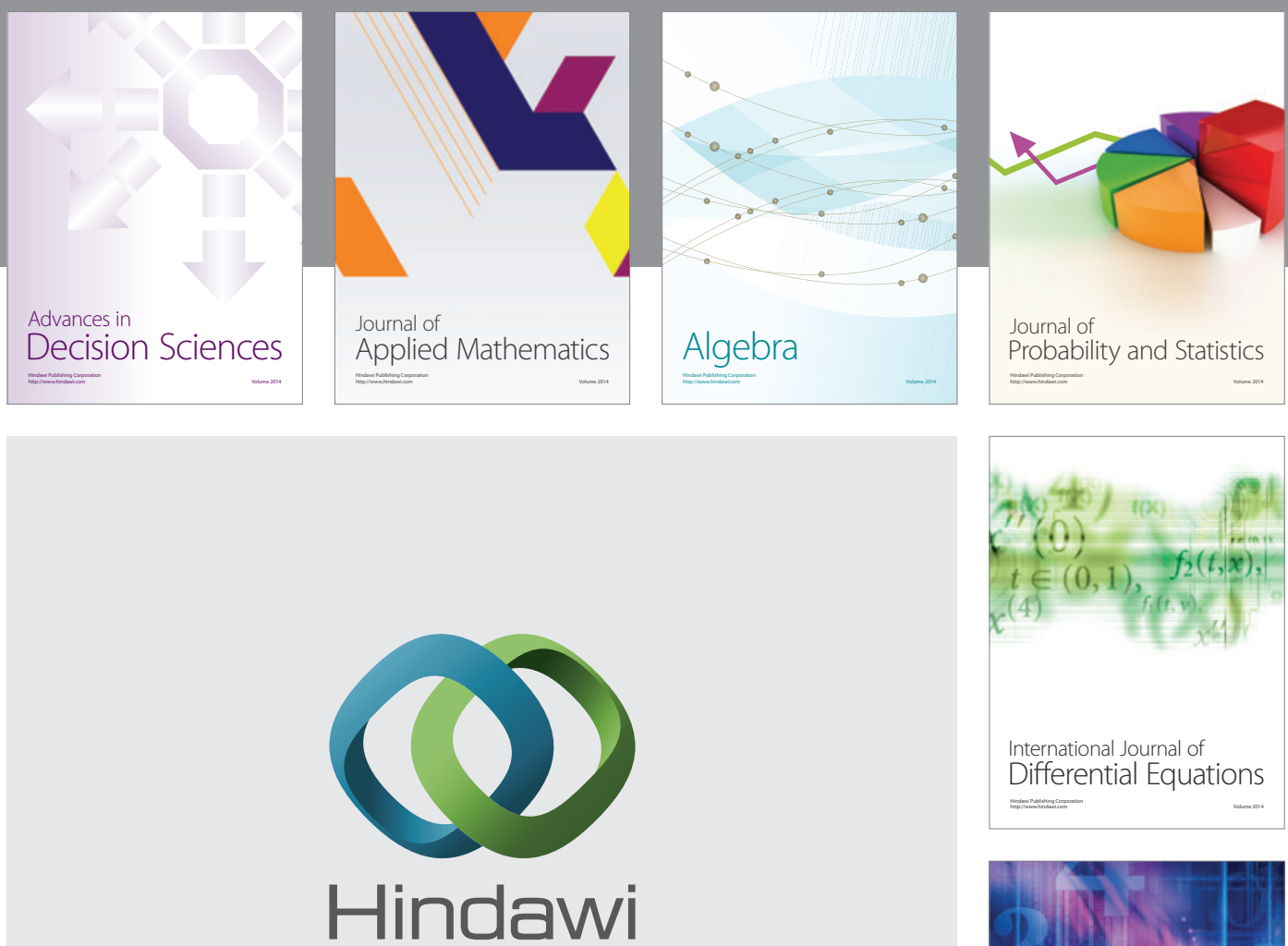

Submit your manuscripts at http://www.hindawi.com
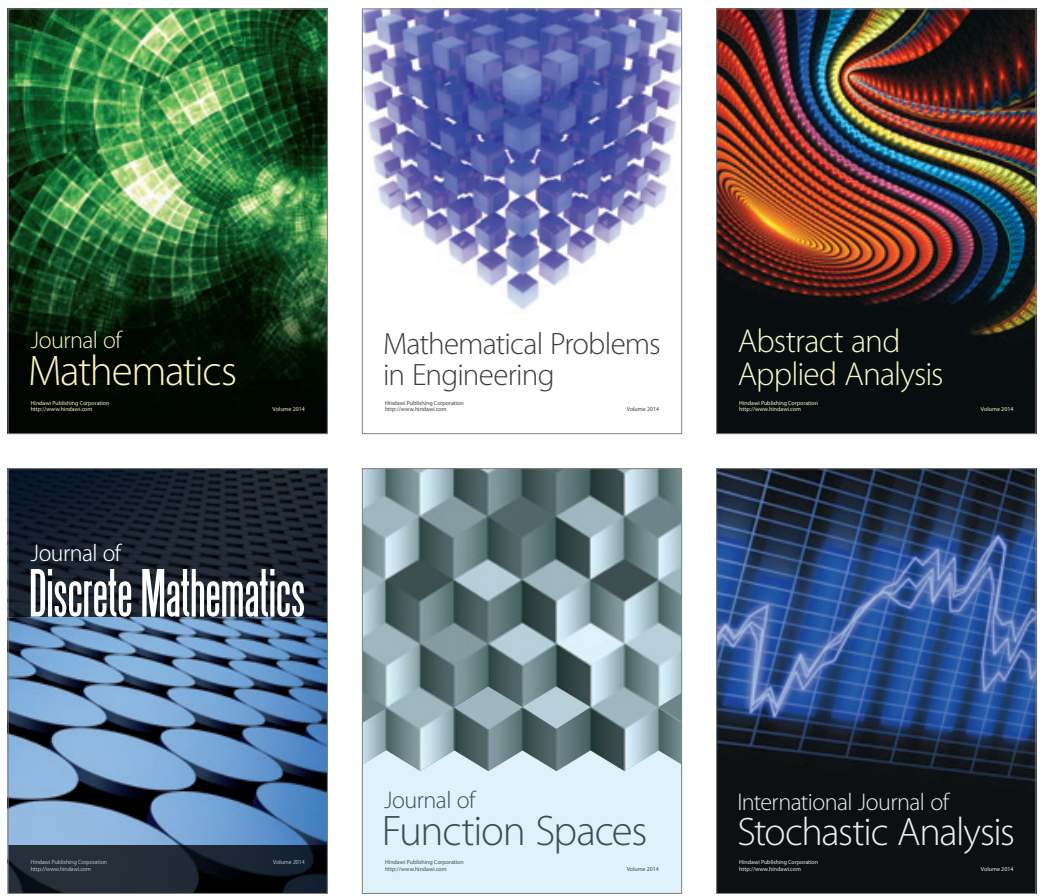

Journal of

Function Spaces



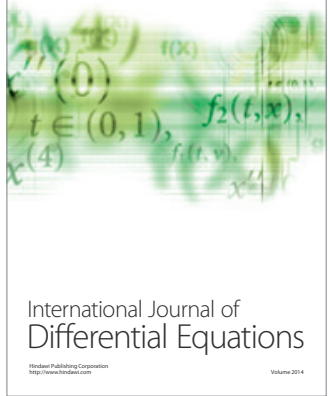
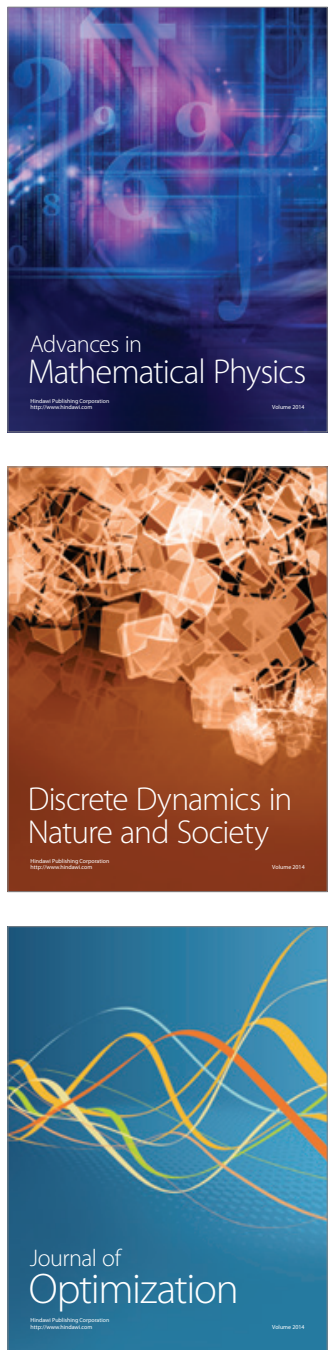